\title{
Descomposición de hojarasca en rodales de Nothofagus pumilio de la región de Magallanes
}

\author{
Litter decomposition in Nothofagus pumilio stands in Magallanes Region
}

\author{
Manuel Ibarra a, Juan Caldentey ${ }^{a *}$, Álvaro Promis ${ }^{a}$ \\ *Autor de correspondencia: ${ }^{a}$ Universidad de Chile, Departamento de Silvicultura y Conservación de la Naturaleza, Santa Rosa \\ 11315, La Pintana, casilla 9206, Santiago, Chile, tel.: 00562978 5721, fax: 00562541 7955, jcaldent@uchile.cl
}

\begin{abstract}
SUMMARY
Litter decomposition in two Nothofagus pumilio stands located in Magallanes Region (Chile) was studied during two years. One stand was a primary forest (SI), without silvicultural treatment, and the other had a regenerative felling under a shelterwood system (CR). Weight loss and litter decomposition rates were obtained with the litter-bag method. At each stand, 36 litter-bags were systematically placed on the forest floor. A fraction of the litter-bags was then randomly extracted at months 2, 5, 9, 11, 12, 15, 21, 22 and 24. The weight of the collected litter-bags was measured at each sampling period. Litter weight-loss presented differences between the stands only after 12 months since the litter-bag set up. After 24 months, the litter weight-loss was similar between the stands, reaching $60 \%$ of the initial weight. After one year of the experimental set-up, litter decomposition rates (k) were 0.76 and 0.44 for CR and SI stands, respectively. At the end of the trial, these values changed to 0.44 and 0.46 for CR and SI stands, respectively. The model that best fitted the relative litter mass according to the initial litter weight was the quadratic regression model, using the number of months, when the litter was on the forest ground, as the independent variable $\left(\mathrm{R}^{2}=0.95\right.$ for both stands). These results suggest that the effect of the regeneration felling on the litter decomposition rates decreases with time.
\end{abstract}

Key words: Nothofagus pumilio, litter decomposition, weight loss, primary forest, regeneration felling.

\section{RESUMEN}

En dos rodales de un bosque lenga (Nothofagus pumilio) de la región de Magallanes, uno sin intervención (SI) y otro sometido a una corta de regeneración (CR), se determinaron las tasas de descomposición de los restos orgánicos (hojarasca) para un período de dos años. En la estimación de las pérdidas de peso y las constantes de descomposición se emplearon 36 bolsas de descomposición dispuestas sistemáticamente en cada rodal, las cuales fueron extraídas a los 2, 5, 9, 11, 12, 15, 21, 22 y 24 meses después de instalado el ensayo. Las pérdidas de peso de la hojarasca mostraron diferencias importantes entre rodales sólo al término del primer año; al finalizar el estudio las pérdidas de peso fueron semejantes, alcanzando valores cercanos al $60 \%$. Las constantes de descomposición (k), transcurrido el primer año, presentaron valores de 0,76 en el rodal con CR y de 0,44 en el SI. Al término del ensayo estos valores llegaron a 0,44 y 0,46 respectivamente. El modelo cuadrático fue el que mejor se ajustó $\left(\mathrm{R}^{2}=0,95\right)$ para estimar la masa relativa de la hojarasca respecto al peso inicial en el tiempo (meses) en ambos rodales. Los resultados obtenidos sugieren que los efectos de la intervención silvicultural sobre las tasas de descomposición de la hojarasca disminuyen con el transcurso del tiempo.

Palabras clave: Nothofagus pumilio, descomposición de la hojarasca, pérdida de peso, corta de regeneración, bosque virgen.

\section{INTRODUCCIÓN}

La descomposición de los residuos orgánicos depositados sobre el piso del bosque constituye una de las vías de mayor importancia en el flujo de nutrientes y en la fertilidad de los ecosistemas forestales. Diversos estudios señalan que las tasas de descomposición de la hojarasca dependen de la interacción de variados factores, entre los que se destacan: los tratamientos silviculturales aplicados a los bosques (Caldentey et al. 2001), las condiciones climáticas y microclimáticas, la cantidad y propiedades físicas y químicas de la hojarasca, así como la abundancia y diversidad de los organismos del suelo (Kochy y Wilson 1997, Berg 2000).
A diferencia de los bosques tropicales, donde la descomposición de los restos orgánicos es bastante rápida, en los ecosistemas de climas templado-fríos este proceso ocurre con mayor lentitud. Por lo mismo, en tales ambientes la dinámica de los aportes y la descomposición de los nutrientes adquieren especial relevancia en el funcionamiento y estabilidad de los bosques, especialmente cuando estos son objeto de intervenciones que remueven parte de su biomasa (Kim et al. 1996).

Responden a dicho contexto, los bosques de lenga (Nothofagus pumilio (Poepp. et Endl.) Krasser) situados en el extremo sur de Sudamérica, que se desarrollan bajo condiciones ambientales severas, con bajas temperaturas, una corta estación de crecimiento y suelos generalmen- 
te ácidos y pobres en nutrientes (Richter y Frangi 1992, Caldentey et al. 2001). Estas formaciones caducifolias subantárticas cubren amplias superficies en la región de Magallanes (Chile), presentando alrededor de 500.000 ha de masas productivas de interés comercial, de las cuales cerca de 30.000 ha se encuentran sometidas al tratamiento silvicultural de cortas de protección, especialmente bajo la intervención de corta de regeneración (Caldentey et al. 2009).

Esta investigación, enmarcada en la temática de evaluar los efectos ambientales de las intervenciones silviculturales aplicadas a los bosques de lenga en la Patagonia chilena y en la hipótesis de que las tasas de descomposición de la hojarasca dependen de la intervención silvicultural y de las modificaciones ocasionadas en el microclima de los rodales, tiene como objetivo estimar dichas tasas, comparando dos rodales: uno sin intervención y otro sometido a una corta de regeneración.

\section{MÉTODOS}

Área de estudio. El estudio se realizó en un bosque de lenga situado en la provincia de Última Esperanza, región de Magallanes, aproximadamente a $180 \mathrm{~km}$ al noroeste de Punta Arenas (52 $05^{\prime}-52^{\circ} 10^{\prime}$ S y $71^{\circ} 35^{\prime}-71^{\circ} 55^{\prime} \mathrm{O}$ ). Este sector se sitúa en el distrito agroclimático de Punta Arenas, presentando un clima marítimo frío (INIA 1989). Los registros meteorológicos obtenidos en los sitios de ensayo (Sánchez 2003) indican que las precipitaciones fluctúan entre 550 y $600 \mathrm{~mm}$ al año; parte importante de esta, especialmente en los meses de julio y agosto, cae en forma de nieve. La temperatura media anual del aire oscila entre 3,8 y $4,1{ }^{\circ} \mathrm{C}$, con máximas medias entre 7,4 y $8,2{ }^{\circ} \mathrm{C}$ y mínimas medias entre 0,5 y $0,7^{\circ} \mathrm{C}$. El período libre de heladas es inferior a un mes, en tanto que la humedad relativa media mensual del aire alcanza entre 83 y $88 \%$.

La topografía predominante corresponde a lomajes suaves, con pendientes promedios menores a $5 \%$. Los suelos, del tipo pardo podzólicos (spodosoles), originados de materiales sedimentarios y volcánicos, normalmente son delgados, de texturas franco arenosas a arenosas, con fertilidad moderada a baja, moderadamente ácidos, sin erosión aparente bajo cubiertas boscosas (Caldentey et al. 2001).

La vegetación natural de esta zona corresponde a la formación del bosque caducifolio de Magallanes, perteneciente a la región del bosque andino patagónico (Gajardo 1994). En ella predominan los bosques puros de lenga en distintos estados de desarrollo, con sotobosque poco diverso y de baja densidad, conformado por Maytenus disticha (Hooker f.) Urban, Pernettya mucronata (L. f.) Gaudich ex G. Don, Ribes magellanicum Poir. y Empetrum rubrum Vahl ex Willd., y un estrato herbáceo escaso, dominado por Osmorhiza chilensis Hooker y Arn.

Rodales estudiados. El estudio fue realizado en dos rodales puros adyacentes de lenga, similares en cuanto a posición fisiográfica y condiciones de sitio; uno correspondía a un bosque natural, sin intervención (SI), y el otro había sido objeto de una corta de regeneración, donde se extrajo aproximadamente el 50 \% de su área basal original (cuadro 1).

Cuadro 1. Características dasométricas de los rodales de Nothofagus pumilio seleccionados en el estudio.

Dendrometric characteristics of the Nothofagus pumilio stands selected in the study.

\begin{tabular}{lcc}
\hline \multirow{2}{*}{ Característica } & \multicolumn{2}{c}{ Rodal } \\
\cline { 2 - 3 } & Sin intervención & $\begin{array}{c}\text { Corta de } \\
\text { regeneración }\end{array}$ \\
\hline $\begin{array}{l}\text { Densidad } \\
\left(\text { árboles ha }{ }^{-1}\right)\end{array}$ & 890 & 213 \\
$\begin{array}{l}\text { Área basal } \\
\left(\mathrm{m}^{2} \text { ha }^{-1}\right)\end{array}$ & 79,3 & 35,8 \\
$\begin{array}{l}\text { Cobertura de copas } \\
(\%)\end{array}$ & 85,3 & 49,2 \\
\hline
\end{tabular}

Descomposición de la hojarasca. En el mes de enero del año 1998, en cada rodal fueron enterradas, sistemáticamente, al nivel del horizonte de descomposición de la hojarasca, 36 bolsas de malla plásticas (litter bag) de $20 \times 30 \mathrm{~cm}$, con un tamaño de poro de $2 \mathrm{~mm}$. En cada malla se puso una cantidad conocida de hojarasca de lenga (30,3 g y 20,2 en rodal sin y con intervención, respectivamente), recolectada en el mismo rodal, pesada en fresco, libre de tierra y con una composición que reflejaba la proporción relativa de los componentes estructurales del material original (hojas, ramillas, cortezas, órganos reproductores y semillas). La concentración inicial de nitrógeno en la hojarasca fue de 0,87 y 0,98 \% en sin y con intervención, respectivamente (Caldentey et al. 2001). Las tasas e índices de descomposición fueron estimados a partir de las pérdidas de peso seco. Para tal efecto, transcurridos 2, 5, 9, 11, 12, 15, 21, 22 y 24 meses desde la instalación del ensayo, se extrajeron tres bolsas de cada rodal. El material obtenido en cada oportunidad fue secado en estufa de aire forzado a $70^{\circ} \mathrm{C}$ hasta un peso constante. Luego se determinó el índice de descomposición "k" de acuerdo a la ecuación 1 (Olson 1963) y su derivación en la ecuación 2.

$$
\begin{aligned}
& \mathrm{X}_{1} / \mathrm{X}_{0}=\mathrm{e}^{-\mathrm{kt}} \\
& \mathrm{k}=-\left(\ln \mathrm{X}_{1} / \mathrm{X}_{0}\right) / \mathrm{t}
\end{aligned}
$$

donde: $\mathrm{X}_{0} \mathrm{y}_{1}$ corresponden al peso seco inicial y final (gramos) del período considerado y t es el tiempo (años).

Análisis de regresión. Un análisis de regresión fue llevado a cabo para estimar la relación entre la masa relativa de hojarasca respecto a su peso inicial y el tiempo en que ella queda en el piso del bosque. Para estos efectos fue utiliza- 
do el algoritmo CURVEFIT de SPSS 15.0 para Windows (SPSS, Cary, NC). Distintos modelos fueron evaluados, tales como funciones lineares, logarítmicas, cuadráticas, exponenciales y de potencia. La bondad de ajuste fue calculada usando el coeficiente de determinación $\left(\mathrm{R}^{2}\right)$, la raíz del error cuadrático medio (RECM) y el valor de significancia $P$ (Sokal y Rohlf 2000).

\section{RESULTADOS}

Descomposición de la hojarasca. Al considerar las pérdidas promedio de peso de la hojarasca, al término del primer año, los porcentajes de descomposición llegaron a 35,6 \% en el rodal sin intervención y a 53,0 \% en el con corta de regeneración. Al finalizar el estudio ( 2 años), estos alcanzaron a 59,7 \% y 58,9\%, respectivamente (cuadro 2).

Si se comparan las variaciones de la masa relativa de la hojarasca de ambos rodales (figura 1) es posible constatar comportamientos similares. Sólo se aprecian diferencias en los valores correspondientes al período comprendido entre el último trimestre del primer año y el primer trimestre del segundo año, en el cual las masas relativas de la hojarasca fueron superiores en el rodal sin intervención.

Al finalizar el primer año, las tasas de descomposición de la hojarasca, evaluadas por el índice "k", fueron de 0,44 y 0,76 para los rodales sin intervención y con corta de regeneración, respectivamente. Transcurridos dos años las diferencias entre rodales fueron mínimas $(\mathrm{k}=0,46 \mathrm{y}$ $0,44)$.

Los mejores modelos de regresión ajustados para la estimación de la descomposición de la hojarasca en ambos rodales dieron como resultado que el 95 \% de la variación en la masa relativa de la hojarasca respecto al peso inicial puede ser estimado a partir del tiempo, en meses, que pasa sobre el piso del bosque, para ambos rodales (cuadro $3 \mathrm{y}$ figura 1).

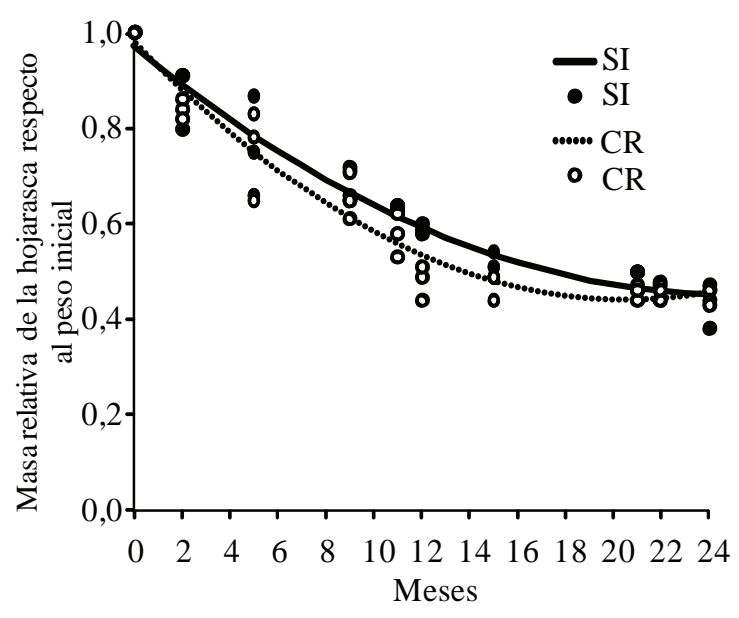

Figura 1. Modelos de regresión ajustados para la estimación de la masa relativa de hojarasca respecto al peso inicial (y) en el tiempo (meses) (x) en que se encuentra en el piso en dos rodales de Nothofagus pumilio (cuadro 3). La línea sólida y los círculos negros corresponden al rodal sin intervención (SI) y la línea punteada y los círculos blancos a la corta de regeneración (CR). Scatter plots of the best relationships to estimate the variations of litter relative masses (y) respect to the time (months) (x) in Nothofagus pumilio stands (table 3 ). Solid line and black circles correspond to the SI stand and the dashed line and white circle to the CR stand.

\section{DISCUSIÓN}

Descomposición de la hojarasca. En ambos rodales la masa remanente de la hojarasca mostró disminuciones relativamente graduales al transcurrir el período de incubación, lo que en términos generales difiere del comportamiento observado en otros estudios sobre descomposición de la hojarasca en bosques templados, donde las pérdidas de peso durante los estados iniciales del proceso usual-

Cuadro 2. Variación del peso seco y de las pérdidas de peso durante la descomposición de la hojarasca en rodales de Nothofagus pumilio sin intervención y con corta de regeneración. Media \pm desviación estándar.

Dry weight and weight loss variation during litter decomposition in Nothofagus pumilio stands without treatment and with regeneration felling. Average \pm standard deviation.

\begin{tabular}{ccccc}
\hline \multirow{2}{*}{$\begin{array}{c}\text { Tiempo } \\
\text { (meses) }\end{array}$} & \multicolumn{2}{c}{ Rodal sin intervención } & \multicolumn{2}{c}{ Rodal con corta de regeneración } \\
\cline { 2 - 5 } & Peso seco $(\mathrm{g})$ & Pérdida peso (\%) & Peso seco (g) & Pérdida peso (\%) \\
\hline 0 & $30,3 \pm 0,0$ & 0,0 & $20,2 \pm 0,0$ & 0,0 \\
2 & $28,8 \pm 1,5$ & 5,0 & $18,9 \pm 0,3$ & 6,4 \\
5 & $25,8 \pm 3,3$ & 14,9 & $17,0 \pm 2,3$ & 15,8 \\
9 & $23,0 \pm 1,6$ & 24,1 & $14,8 \pm 1,4$ & 26,7 \\
11 & $21,4 \pm 0,2$ & 29,4 & $12,5 \pm 1,5$ & 38,1 \\
\hline 12 & $19,5 \pm 0,4$ & 35,6 & $9,5 \pm 1,1$ & 53,0 \\
15 & $15,8 \pm 1,1$ & 47,9 & $9,2 \pm 0,9$ & 54,5 \\
21 & $14,6 \pm 1,1$ & 51,8 & $8,7 \pm 0,5$ & 56,9 \\
22 & $13,2 \pm 1,1$ & 56,4 & $8,6 \pm 0,5$ & 57,4 \\
24 & $12,2 \pm 2,1$ & 59,7 & $8,3 \pm 0,5$ & 58,9 \\
\hline
\end{tabular}


Cuadro 3. Mejores modelos de regresión ajustados para la estimación de la masa relativa de hojarasca respecto al peso inicial (y) en el tiempo (meses) (X) en que se encuentra en el piso en dos rodales de Nothofagus pumilio, un rodal sin intervención y otro sometido a corta de regeneración. $\mathrm{b}_{0}, \mathrm{~b}_{1}, \mathrm{~b}_{2}=$ coeficientes del modelo, $\mathrm{R}^{2}=$ coeficiente de determinación, $\mathrm{RECM}=$ raíz del error cuadrático medio ( $\mathrm{n}=30$ observaciones).

Results of the best model fitted to estimate the relative litter mass respect to the initial litter weight (y) based on the months (X) during which the litter is on the forest floor, as the independent variable in Nothofagus pumilio stands without treatment and with regeneration felling. $\mathrm{b}_{0}$, $\mathrm{b}_{1}$, $\mathrm{b}_{2}$ are the model coefficients, $\mathrm{R}^{2}$ is the coefficient of determination, $\mathrm{RECM}$ is the root mean square error (number of observations $=30$ ).

\begin{tabular}{lccccccc}
\hline Rodal & Modelo & $\mathrm{b}_{0}$ & $\mathrm{~b}_{1}$ & $\mathrm{~b}_{2}$ & $\mathrm{R}^{2}$ & $\mathrm{RECM}$ & $P$ \\
\hline Sin intervención & $\mathrm{y}=\mathrm{b}_{0}+\mathrm{b}_{1} \mathrm{X}+\mathrm{b}_{2} \mathrm{X}^{2}$ & 0,974 & $-0,042$ & 0,001 & 0,946 & 0,044 & 0,000 \\
Corta de regeneración & $\mathrm{y}=\mathrm{b}_{0}+\mathrm{b}_{1} \mathrm{X}+\mathrm{b}_{2} \mathrm{X}^{2}$ & 0,978 & $-0,052$ & 0,001 & 0,945 & 0,046 & 0,000 \\
\hline
\end{tabular}

mente son mucho más marcadas (Guillon et al. 1994). Tal comportamiento podría atribuirse a que el sustrato de descomposición utilizado, a diferencia de las evaluaciones realizadas en la mayoría de los estudios, consideró todos los componentes de la hojarasca, incluyendo tanto las estructuras de descomposición rápida (hojas y elementos reproductivos), como las de lenta descomposición (ramillas y corteza).

Las pérdidas promedio de peso seco mostraron diferencias importantes entre rodales sólo hacia el término del primer año, alcanzando valores de 35,6 \% bajo el rodal sin intervención y de 53,0 \% en el con corta de regeneración. Transcurridos dos años, sin embargo, las pérdidas de peso fueron muy similares, con valores cercanos al $60 \%$ en los dos rodales. Las magnitudes alcanzadas por la masa remanente se sitúan dentro del rango señalado para especies de diferentes tipos de bosque y condiciones climáticas (Guillon et al. 1994, Alhamd et al. 2004). Al respecto, Prescott et al. (2000) reportan pérdidas de masa que variaban entre 53 y 75 \% para la hojarasca de Populus sp. de diferentes sitios en British Columbia. A su vez, Sariyildiz y Anderson (2003), señalan que transcurridos doce meses las pérdidas de masa de la hojarasca de Fagus sylvatica L. fueron de 25,5-27,8 \%. Para la misma especie, pero en ambientes mediterráneos, Cortez (1998) determinó que después de dos años de iniciada la descomposición las pérdidas de masa variaban entre 38,5 y 61,7 \%, dependiendo de las condiciones del sitio analizado, concluyendo que en sitios con menor actividad de las comunidades de gusanos de tierra, la descomposición estaba influida de modo más importante por las condiciones climáticas (temperatura y humedad).

Las constantes de descomposición " $k$ " obtenidas al cabo de dos años de iniciado el estudio fueron muy similares, con valores de 0,46 en el rodal sin intervención y de 0,44 en el con corta de regeneración. Las variaciones respecto a lo registrado al término del primer año fueron mínimas para la hojarasca del rodal sin intervención $(0,44)$ y bastante marcadas en el caso de la corta de regeneración, donde se redujo de 0,76 a 0,44. En términos generales, las constantes de descomposición corresponden a los valores típicos de los bosques templados $(0,21-0,57)$ (Laskowski et al. 1995). De modo más específico, los valores de "k", transcurridos dos años de iniciada la descomposición, se sitúan en torno al rango inferior determinado por Richter y Frangi (1992) en bosques de lenga del sur de Argentina (0,47-0,76).

De acuerdo a los resultados obtenidos, el efecto de la intervención silvicultural sobre la descomposición de la hojarasca (corta de regeneración) no sería permanente en el tiempo y sólo marcaría diferencias de importancia hacia fines del primer año de descomposición. Se ha señalado que el tipo de especie puede afectar las tasas de descomposición tanto a través de la calidad o masa de hojarasca, la acción del microclima o las comunidades de descomponedores presentes (Kochy y Wilson 1997). Puesto que los dos rodales estudiados presentaban condiciones iniciales muy similares en cuanto a composición de especies, estado de desarrollo y condiciones de sitio, las diferencias iniciales en la velocidad de descomposición observadas podrían, en principio, ser atribuidas a los cambios microclimáticos ocasionados por la intervención (Promis et al. 2010). Aunque la importancia relativa del microclima sobre la descomposición de la hojarasca ha sido poco estudiada a escala local (Kochy y Wilson 1997), se estima que las variaciones en los factores microclimáticos afectarían potencialmente las tasas de descomposición. En tal sentido, diferencias en el sombreamiento debidas a variaciones en la cobertura del dosel, con sus efectos sobre la insolación, la temperatura y la humedad del suelo estarían influyendo en las tasas de descomposición (Kochy y Wilson 1997). Así, el mayor sombreamiento del rodal no intervenido, con valores significativamente inferiores de radiación total, $76 \%$ en fase de crecimiento vegetativo y 71,7 \% en fase de receso vegetativo (Promis et al. 2010), podría asociarse a menores velocidades de descomposición que en el rodal con corta de regeneración. Además, los contenidos de humedad de este último no habrían constituido una limitante, toda vez que la precipitación neta que recibe este rodal ha sido claramente superior (Promis 1999).

No obstante lo anterior, estudios realizados en diferentes especies muestran resultados variables en las tasas de descomposición de la hojarasca respecto a las intervenciones del dosel arbóreo. Así, Kim et al. (1996) al evaluar 
los efectos de cuatro tratamientos al dosel (tala rasa, coberturas de 50 y $75 \%$ y sin intervención), en bosques de Quercus rubra L., determinaron que las pérdidas de peso de la hojarasca, al cabo de dos años, fueron significativamente superiores en el sector sometido a tala rasa. Prescott (1997), en cambio, al comparar bosques maduros de Tsuga sp. y Abies sp. con sectores adyacentes sometidos a distintos tipos de cosecha encontró que, transcurridos dos años, las pérdidas de masa de la hojarasca fueron más rápidas en el bosque antiguo, no intervenido; efecto que fue atribuido a la mayor humedad del suelo superficial durante el verano. En el caso de rodales del género Populus se ha reportado que las pérdidas de peso en bosques naturales y en sectores sometidos a tala rasa son de similar magnitud (Prescott et al. 2000). Por tal razón, Prescott (2005) señala que las tasas de descomposición no constituirían parte integral de las respuestas de los ecosistemas forestales a factores como la tala.

Por otra parte, Salamanca et al. (2003), al analizar el efecto de las precipitaciones en la descomposición de la hojarasca de Quercus sp., encontraron que las pérdidas de peso fueron significativamente menores en el tratamiento donde se redujo totalmente el aporte de las precipitaciones respecto al que se dejó totalmente expuesto. La mayor tasa de descomposición de este último fue atribuida al efecto de las precipitaciones junto a la acción de los microbios y la fauna del suelo.

Además del probable efecto del microclima en las tasas iniciales de descomposición, también podrían haber determinado algunas diferencias en las condiciones del suelo mineral, como los mayores valores de $\mathrm{pH}$ y concentraciones de nitrógeno, fósforo y potasio disponibles detectados en el rodal con corta de regeneración (Caldentey et al. 2001). Al respecto, Sariyildiz y Anderson (2003) reportan que las pérdidas de masa para $F$. sylvatica, a los 12 meses, fueron superiores en sitios con mayores contenidos de nutrientes, coincidiendo con lo publicado en otros estudios (Keenan et al. 1996).

Otro factor que podría haber incidido en las fases iniciales de la descomposición sería las diferencias en la concentración inicial de nitrógeno en la hojarasca, las que según Caldentey et al. (2001) correspondieron a 0,87 y $0,98 \%$ del peso seco de la hojarasca en los rodales sin y con intervención, respectivamente. Al respecto, diferentes estudios coinciden en señalar que el contenido de nutrientes en las hojas también afecta las tasas de descomposición. Así, por lo general, altos niveles de nutrientes, especialmente nitrógeno, serían capaces de acelerar el proceso de descomposición (Mfilinge et al. 2002). En el mismo sentido, se ha indicado que dentro de un mismo hábitat, los índices de calidad de la hojarasca, basados en sus contenidos de nitrógeno, son los mejores predictores de las tasas de descomposición. Así, en restos orgánicos con altas concentraciones de nitrógeno la descomposición sería más rápida que en residuos con bajas concentraciones a igual contenido de lignina (Fog 1988).
Rutigliano et al. (1996), en tanto, señalan que la concentración de nitrógeno no influiría ni en el estado inicial ni final de la descomposición de la hojarasca de F. sylvatica, y que las pérdidas de peso estarían más influenciadas por las concentraciones de lignina. En sus resultados muestran que a menores concentraciones de lignina más altas fueron las pérdidas de peso y que la degradación de este compuesto comienza conjuntamente con el inicio de la incubación. También la concentración inicial de sustancias solubles ha sido relacionada con las tasas de pérdidas iniciales de masa (Guillon et al. 1994). Al respecto, Alexander y Beat (2004) determinaron que las pérdidas de peso de la hojarasca de F. sylvatica tomadas en un transecto altitudinal de bosques suizos, el cual incluía diversos tipos de suelo, estuvieron mejor correlacionadas con el contenido de sustancias solubles. Además, indican una pequeña influencia del clima al comienzo del proceso de descomposición y una fuerte influencia de la calidad del sustrato durante los dos primeros años de éste.

El comportamiento temporal de la descomposición de la hojarasca de lenga, en general, mostró cambios relativamente graduales. Aun así, es posible advertir mayores velocidades de descomposición al término del primer año (corta de regeneración) o a comienzos del segundo (sin intervención). Estos resultados se aproximan a lo señalado por diversos autores, en el sentido que durante la descomposición es posible reconocer dos estados (Alhamd et al. 2004). En la fase inicial, las concentraciones de las sustancias solubles en agua decrecen rápidamente (Osono y Takeda 2001) y parte de la holocelulosa es degradada, mientras la lignina recalcitrante no se descompone o sólo lo hace en muy baja proporción. De este modo la concentración de la lignina comienza a incrementarse debido a la descomposición de los otros componentes. También la concentración de algunos nutrientes como el nitrógeno, fósforo y azufre comienza a aumentar (Staff y Berg 1982).

En los estados tardíos, la descomposición de la lignina y las celulosas lignificadas dominarían sobre la influencia de los nutrientes regulando así la descomposición de la hojarasca (Osono y Takeda 2001). Diversos autores refieren una disminución en las tasas de descomposición para los estados más tardíos del proceso, estimándose tasas extremadamente lentas para los estados finales (Guillon et al. 1994). En tales casos, las pérdidas acumuladas de peso se aproximarían a un valor límite de descomposición, el cual es descrito como una función asintótica (Berg 2000). Al respecto, Berg et al. (1996) han estimado valores límites de descomposición entre 50-65 \% para Fagus grandifolia Ehrh., valores que corresponden a lo observado en este estudio.

Los aspectos antes señalados podrían explicar el hecho que las tasas de descomposición encontradas sean más lentas hacia fines del segundo año y que, con el tiempo, tiendan a hacerse semejantes en las dos situaciones consideradas, independientemente de la condición microclimática y de las características iniciales del suelo y de la hojarasca. 


\section{CONCLUSIONES}

Los resultados obtenidos permiten asumir que la intervención silvicultural realizada (corta de regeneración) sólo tendría efectos importantes en las tasas iniciales de descomposición, debido a los cambios microclimáticos ocasionados por la reducción en la cobertura del dosel y, en cierta medida, por las diferencias en los contenidos iniciales de nutrientes en la hojarasca. Con el transcurso del tiempo, sin embargo, los efectos de la intervención van disminuyendo, lo que sugiere una mayor incidencia de las propiedades intrínsecas del material utilizado o la probable predominancia de otros factores, hechos que no fueron determinados en este estudio. De todas maneras, para poder indicar los reales efectos de las cortas de regeneración sobre las tasas de descomposición de la hojarasca es necesario llevar a cabo más investigaciones, en las que se pueda incorporar una mayor cantidad de repeticiones (por ejemplo, considerando diferentes pendientes, porcentajes de cosecha, época de cosecha, fase de desarrollo de los rodales) y en los que se pueda relacionar esta información con antecedentes microclimáticos y diferentes contenidos de nutrientes iniciales.

\section{AGRADECIMIENTOS}

El estudio fue financiado con aportes del Fondo Nacional de Investigaciones Científicas y Tecnológicas (FONDECYT) a través del Proyecto $\mathrm{N}^{\circ}$ 1960936. Al mismo tiempo agradecemos el apoyo logístico y el trabajo de campo realizado por el Ingeniero Forestal Marcelo Álvarez y otros colaboradores de la Corporación Nacional Forestal.

\section{REFERENCIAS}

Alexander H, F Beat. 2004. Early stage litter decomposition rates for Swiss forests. Biogeochemistry 70: 299-313.

Alhamd L, S Arakaki, A Hagihara. 2004. Decomposition of leaf litter of four tree species in a subtropical evergreen broadleaved forest, Okinawa Island, Japan. Forest Ecology and Management 202: 1-11.

Berg B. 2000. Litter decomposition and organic matter turnover in northern forest soils. Forest Ecology and Management 133: 13-22.

Berg B, G Ekbohm, M Johansson, C Mc Claugherty, F Rutigliano, A Desanto. 1996. Maximum decomposition limits of forest litter types: a synthesis. Canadian Journal of Botany 74: 659-672.

Caldentey J, M Ibarra, J Hernández. 2001. Litter fluxes and decomposition in Nothofagus pumilio stands in the región of Magallanes, Chile. Forest Ecology and Management 148: 145-157.

Caldentey J, H Mayer, M Ibarra, A Promis. 2009. The effects of a regeneration felling on photosynthetic photon flux density and regeneration growth in a Nothofagus pumilio forest. European Journal of Forest Research 128: 75-84.

Cortez J. 1998. Field decomposition of leaf litters relationships between decomposition rates and soil moisture, soil temperature and earthworm activity. Soil Biology and Biochemistry 30: 783-793.

Fog K. 1988. The effect of added nitrogen on the rate of decomposition of organic matter. Biological Reviews 63: 433-462.

Gajardo R. 1994. La vegetación natural de Chile. Clasificación y distribución geográfica. Santiago, Chile. Editorial Universitaria. 165 p.

Guillon D, R Jofré, A Ibrahima. 1994. Initial litter properties and decay-rate - a microcosm experiment on mediterranean species. Canadian Journal of Botany 72: 946-954.

INIA (Instituto Nacional de Investigaciones Agropecuarias, CL). 1989. Mapa Agroclimático de Chile. Santiago, Chile. Ministerio de Agricultura, Instituto Nacional de Investigaciones Agropecuarias. $221 \mathrm{p}$.

Keenan R, C Prescott, J Kimmins, J Pastor, B Dewey. 1996. Litter decomposition in western hemlock forest on northern Vancouver Island, British Columbia. Canadian Journal of Botany 74: 1626-1634.

Kim C, T Sharik, M Jurgensen. 1996. Canopy cover effects on mass loss, and nitrogen and phosphorus dynamics from decomposing litter in oak and pine stands in northern Lower Michigan. Forest Ecology and Management 80: 13-20.

Kochy M, S Wilson. 1997. Litter decomposition and nitrogen dynamics in aspen forest and mixed-grass prairie. Ecology 78: 732-739.

Laskowski R, M Niklinska, M Maryanski. 1995. The dynamics of chemical elements in forest litter. Ecology 76: 1393-1406.

Mfilinge P, N Atta, M Tsuchiya. 2002. Nutrient dynamics and leaf litter decomposition in a subtropical mangrove forest at Oura Bay, Okinawa. Japan. Trees 16: 172-180.

Olson J. 1963. Energy storage and the balance of producers and decomposers in ecological systems. Ecology 44: 322-331.

Osono T, H Takeda. 2001. Organic chemical and nutrient dynamics in decomposing beech leaf litter in relation to fungal ingrowth and succession during 3-year decomposition processes in a cool temperate deciduous forest in Japan. Ecological Research 16: 649-670.

Prescott C.1997. Effects of clearcutting and alternative silvicultural systems on rates of decomposition and nitrogen mineralization in a coastal montane coniferous forest. Forest Ecology and Management 95: 253-260.

Prescott C. 2005. Do rates of litter decomposition tell us anything we really need to know? Forest Ecology and Management 220: 66-74.

Prescott C, L Blevins, C Staley. 2000. Effects of clear-cutting on decomposition rates of litter and forest floor in forests of British Columbia. Canadian Journal of Forest Research 30: 1751-1757.

Promis A. 1999. Modificaciones microclimáticas causadas por la intervención silvícola en bosques naturales de lenga (Nothofagus pumilio). Memoria Ingeniero Forestal. Santiago, Chile. Universidad de Chile, Facultad de Ciencias Forestales. 108 p.

Promis A, J Caldentey, M Ibarra. 2010. Microclima en el interior de un bosque de Nothofagus pumilio y el efecto de una corta de regeneración. Bosque 31: 129-139.

Richter L, J Frangi. 1992. Bases ecológicas para el manejo del bosque de Nothofagus pumilio de Tierra del Fuego. Revista de la Facultad de Agronomía, La Plata 68: 35-52.

Rutigliano F, A Virzo de Santo, B Berg, A Alfani, A Fioretto. 1996. Lignin decomposition in decaying leaves of Fagus 
sylvatica L. and needles of Abies alba Mill. Soil Biology and Biochemistry 28: 101-106.

Salamanca E, N Kaneko, S Katagiri. 2003. Rainfall manipulation effects on litter decomposition and the microbial biomass of the forest floor. Appied Soil Ecology 22: 271-281.

Sánchez P. 2003. Cambios microclimáticos debido a cortas de protección y su influencia en el desarrollo de la regeneración natural de Lenga (Nothofagus pumilio (Poepp. et Endl.) Krasser). Memoria Ingeniero Forestal. Santiago, Chile. Universidad de Chile, Facultad de Ciencias Forestales. 69 p.
Sariyildiz T, J Anderson. 2003. Interactions between litter quality, decomposition and soil fertility: a laboratory study. Soil Biology and Biochemistry 35: 391-399.

Sokal RR, FJ Rohlf. 2000. Biometry. The principles and practice of statistics in biological research, 3rd ed. New York, USA. Freeman and Company. 863 p.

Staff H, B Berg. 1982. Accumulation and release of plant nutrients in decomposing Scots pine needle litter. II. Longterm decomposition in a Scots pine forest. Canadian Journal of Botany 60: 1561-1568.

Recibido: 30.09.10

Aceptado: 21.07.11 
